\title{
A resolutividade em saúde bucal na atenção básica como instrumento para avaliação dos modelos de atenção
}

\author{
Resolubility in oral health for primary care \\ as an instrument for the evaluation of health systems
}

Jaqueline Bulgareli $^{1}$

Karine Laura Cortellazzi ${ }^{1}$

Glaucia Maria Bovi Ambrosano ${ }^{1}$

Marcelo de Castro Meneghim ${ }^{1}$

Eduardo Tanajura de Faria ${ }^{2}$

Fábio Luiz Mialhe ${ }^{1}$

Antonio Carlos Pereira ${ }^{1}$

' Faculdade de Odontologia de Piracicaba, Unicamp. Avenida Limeira 901, Areião. 13.414-903 Piracicaba SP Brasil. jaquelinebulgareli@gmail.com ${ }^{2}$ Secretaria Municipal de Saúde de Marília.
Abstract This study sought to evaluate which health system - either the Family Health Strategy (FHS) or the traditional model established in the Basic Health Units (BHU) - was more effective during the period between 2007 and 2009 in relation to the oral health needs of the population of the city of Marilia in the state of São Paulo. It involved a retrospective ecological study with a stratified random sample of $50 \%$ of the universe of the study area for the $12 \mathrm{BHU}$ and $37 \%$ of the 29 Family Health Units (FHU) in the municipality, whereby 6 BHU and 11 FHS were randomly selected. The data collected for observation were gathered through the management reports of the SIAB and Oral Health Assessment Report information systems. Exploratory data analysis and the Student $t$ test were performed in order to analyze the resolubility of different systems of health care. It was observed that the coefficients relating to Initial Dental Treatment were not statistically different, though with respect to the Completed Initial Treatment coefficient for the population greater than two years the values were 16.74 for the FHS model and 6.93 for the BHU model, with a statistically significant difference of $40.97 \%$ resolubility for the BHU in relation to $83.56 \%$ for the FHS $(p<0.05)$.

Key words Evaluation of health systems, Oral health, Primary care, Resolubility
Resumo Este estudo teve como objetivo avaliar qual modelo de atenção, Estratégia Saúde da Família (ESF) ou modelo tradicional instituído nas Unidades Básicas de Saúde (UBS) apresenta maior efetividade em relação às necessidades em saúde bucal da população de Marília (SP) durante o período de 2007 a 2009. Estudo ecológico do tipo retrospectivo com uma amostra aleatória estratificada de 50\% do universo da área de estudo para as 12 UBS e 37\% das 29 Unidades de Saúde da Família (USF) do município, sendo sorteadas aleatoriamente 6 UBS e 11 USF, respectivamente. Os dados coletados para observação foram levantados através dos relatórios gerenciais dos sistemas de informação, SIAB e Relatório de Avaliação de Saúde Bucal. Para analisar a resolutividade dos diferentes modelos de atenção à saúde, realizou-se análise exploratória dos dados e o teste tde Student. Observou-se que os coeficientes relativos ao Tratamento Odontológico Inicial não foram estatisticamente diferentes. Já no que se refere ao coeficiente Tratamento Completado Inicial sobre a população estimada maior que dois anos, verificaram-se valores de 16,74 para o modelo USF e 6,93 para o modelo UBS, havendo uma diferença estatisticamente significativa de $40,97 \%$ de resolutividade na UBS em relação aos $83,56 \%$ da USF.

Palavras-chave Avaliação de serviços de saúde, Saúde bucal, Atenção primária, Resolutividade 


\section{Introdução}

A criação do Sistema Único de Saúde (SUS), em 1988, incorpora na política de saúde do Brasil os princípios da universalidade, equidade e integralidade, com as seguintes características: existência de uma integração na relação profissionalusuário, abrangendo a totalidade psicofisiológica e social, como um todo indivisível, inserido numa organização institucional que englobe ações de promoção, proteção e recuperação da saúde ${ }^{1}$.

Nesse momento, os olhares se voltam para o reordenamento do sistema de saúde brasileiro, redefinindo a lógica de acesso dos usuários aos serviços de saúde e o papel da Atenção Primária à Saúde (APS), em especial na "porta de entrada" e na coordenação da rede de atenção. Tal pensamento se deve ao entendimento de que a sustentabilidade dos sistemas de saúde, baseados nos modelos médico-hospitalocêntricos, apresentava evidente sinal de esgotamento, com questionamento devido à cultura da busca pelo especialista e da livre demanda, onde vários países que restringem o acesso a especialistas ao encaminhamento do médico da APS apresentam repercussões positivas nos custos e na eficiência do sistema ${ }^{2}$.

A APS é caracterizada por um conjunto de ações, no âmbito individual e coletivo, que abrangem a promoção e a proteção da saúde, a prevenção de agravos, o diagnóstico, o tratamento, a reabilitação e a manutenção da saúde ${ }^{3}$, sendo demonstrada na literatura como elemento-chave, com capacidade de influir nos indicadores e com grande potencial regulador da utilização de recursos tecnológicos de alta densidade tecnológica, garantindo o acesso universal aos serviços que tragam reais benefícios à população ${ }^{1}$.

Em 1994, foi implantado o Programa Saúde da Família (PSF), atualmente denominado Estratégia Saúde da Família (ESF). As equipes de Saúde da Família buscam uma nova prática assistencial que seja integral na atenção às necessidades em saúde ${ }^{4}$, visando à resolução da maioria dos problemas e necessidades dos indivíduos, famílias e comunidade de um território definido, conforme descrito pela Organização Mundial da Saúde. Esse serviço, que é porta de entrada do sistema de saúde, deveria ser capaz de resolver pelo menos $80 \%$ dos problemas ${ }^{5}$, ou até $85 \%$ da necessidades, realizando serviços preventivos, curativos, reabilitadores e de promoção ${ }^{6}$.

Desta forma, no município de Marília existem diferentes arranjos organizacionais de APS com vistas à efetividade da resolução da grande maioria dos problemas e necessidades de saúde da população. Assim, antes da implantação da Saúde da Família, as unidades que desenvolviam assistência no nível de atenção básica atuavam com vistas à cura de doenças, onde a organização do processo de trabalho priorizava a demanda espontânea, ou seja, práticas tradicionais de atenção ${ }^{7}$, as quais ainda permanecem instituídas na vasta rede de atenção básica onde não se encontra a Saúde da Família implantada, principalmente no Estado de São Paulo, um dos estados com menor cobertura populacional de Saúde da Família no Brasil.

Considerando o processo histórico da saúde bucal no Brasil, marcada pela deficiente assistência odontológica pública causando superlotação dos postos de atendimento odontológico e grande limitação do acesso da população aos serviços prestados, se fazia necessária a construção de uma Política Nacional de Saúde Bucal, a qual foi editada e vem sendo incentivada pelo Governo Federal desde o final de 2003 com a intitulação: Programa Brasil Sorridente ${ }^{8}$. Esta política apresenta diretrizes que apontam para a ampliação e a qualificação do atendimento em saúde bucal na atenção básica, através da realização de atividades de promoção, prevenção, assistência e reabilitação, possibilitando o acesso a todas as faixas etárias ${ }^{3}$.

Entretanto, apesar da expansão do número de ESB, questiona-se, atualmente, se houve aumento quantitativo ou mesmo alguma mudança nos tipos de procedimentos oferecidos à população com a introdução da ESB na ESF, pois se observa que a atenção à saúde bucal no Brasil tem-se caracterizado pela insuficiência de procedimentos coletivos e preventivos individuais e pela baixa cobertura de procedimentos curativos e de urgência.

Desta forma, faz-se necessário a realização de estudos que efetivamente demonstrem os resultados e o impacto das ações odontológicas realizadas pelas ESB na ESF em relação às ações desenvolvidas por outros arranjos organizacionais dos serviços de atenção básica a exemplo do modelo tradicional ainda existente na maioria dos municípios paulistas, realizando o monitoramento e a avaliação das ações desenvolvidas em saúde bucal, através de indicadores que nos permitem elaborar um conhecimento sobre uma determinada situação?.

Além dos indicadores do Ministério da Saúde é importante ressaltar que cada município, conforme suas necessidades e peculiaridades, deve discutir junto à população e aos profissionais de saúde bucal outros indicadores com vistas à melhoria na população ${ }^{10}$. 
Conforme a Portaria GM/MS 3.925/98 ${ }^{11}$, resolutividade pode ser entendida ainda como a eficiência na capacidade de resolução das ações e serviços de saúde, através da assistência integral resolutiva, contínua e de boa qualidade à população adstrita, no domicílio e na unidade de saúde, buscando identificar e intervir sobre as causas e os fatores de risco aos quais essa população está exposta ${ }^{12}$.

Contudo não há na literatura nenhum artigo publicado sobre a questão da resolutividade de ações em saúde bucal na atenção básica, principalmente, levando em consideração os diferentes modelos de gestão utilizados. Desta forma, este estudo tem como objetivo avaliar a resolutividade das ações de saúde bucal desenvolvidas na atenção básica do município de Marília (SP), através dos indicadores de acesso ao tratamento odontológico e o percentual de cobertura populacional em Tratamento Completado Inicial (TCI), com vistas à identificação de qual modelo de atenção, ESF ou modelo tradicional instituído nas UBS, apresenta maior efetividade em relação às necessidades em saúde bucal da população.

\section{Material e método}

Marília é uma cidade localizada no centro-oeste paulista, fica distante da capital $438 \mathrm{~km}$. Ocupa uma área de $1.170 \mathrm{Km}^{2}$ e conta com uma população de aproximadamente 218.228 mil habitantes ${ }^{13}$, sendo assim a $32^{\text {a }}$ maior do estado e a décimo quarta do interior paulista em número de habitantes. O IDH é de 0,821 .

Trata-se de um estudo ecológico do tipo retrospectivo, para análise da resolutividade das ações de saúde bucal desenvolvidas na atenção básica. A amostra foi aleatória estratificada com $50 \%$ do universo da área de estudo para as 12 Unidades Básicas de Saúde (UBS) e 37\% das 29 Unidades de Saúde da Família (USF), portanto 6 UBS e 11 USF, selecionadas aleatoriamente por região de saúde de Marília (SP).

Atualmente, a rede de atenção básica à saúde bucal do município está distribuída em 12 Unidades Básicas de Saúde (cobertura de 53,9\%), 33 Unidades de Saúde da Família $(46,1 \%)$ e 3 Pronto Atendimentos. Existe também assistência odontológica aos pacientes institucionalizados que frequentam as associações filantrópicas: Legião Mirim, Educandário Bento de Abreu Sampaio Vidal, Associação de Combate ao Câncer (ACC) e Hospital Espírita de Marília (HEM).

No município de Marília é utilizado o indicador TCI em Odontologia, que se refere à conclu- são dos procedimentos odontológicos compreendidos no âmbito da atenção básica, de um usuário com idade superior a dois anos que apresenta necessidades curativas. O TCI é acompanhado pelo cirurgião-dentista por até dois anos, sendo que após este período, se o usuário apresentar novas necessidades odontológicas curativas deverá submeter-se a novo TCI, podendo este identificar a relação de tratamentos odontológicos que foram iniciados e completamente terminados.

Na Unidade Básica de Saúde todos os CD têm vínculo direto do tipo estatutário com a Prefeitura Municipal de Marília cumprindo carga horária de 20 horas, sendo que para cada unidade existem dois profissionais que atuam em períodos distintos (matutino e vespertino). Neste modelo, o profissional cirurgião-dentista trabalhou na lógica de "duas mãos", sem o profissional auxiliar em saúde bucal (ASB).

A organização do processo de trabalho na UBS é estruturada na assistência individual, atendimento de urgência, tratamento curativo e preventivo e reunião de equipe mensal, da qual nem sempre o CD participa. A grande maioria dos pacientes é agendada por livre demanda durante o horário de acolhimento da unidade de saúde, através de blocos de consulta (grupo de pacientes agendados no mesmo horário).

No município de Marília, a Saúde da Família foi implantada em 1998, com a equipe de saúde bucal já incorporada ao quadro de profissionais da equipe mínima, em todas as unidades.

Na unidade de Saúde da Família, todos os CD possuem vínculo pela Consolidação das Leis Trabalhistas - CLT com entidade filantrópica do município (Associação Feminina de Marília Maternidade e Gota de Leite) que desenvolve parceria, por meio de convênio, com a Prefeitura $\mathrm{Mu}$ nicipal de Marília cumprindo 40 horas semanais e atuam no período matutino e vespertino em uma única unidade. Neste modelo o profissional cirurgião-dentista trabalha na lógica de "quatro mãos", com o ASB compondo uma equipe de Saúde Bucal - (ESB) modalidade I (um dentista + um ASB), conforme portaria GM/MS no 648/ $2006^{14}$.

Na Saúde da Família a assistência é familiar, realiza a visita domiciliar e a busca ativa dos pacientes faltosos tanto nas consultas programáticas da atenção básica, como no atendimento especializado. Os pacientes eletivos são atendidos com horário programado. Já os pacientes que procuram o serviço com demanda espontânea, recebem atendimento no início dos períodos de trabalho de acordo com a disponibilidade de 
vagas, exceto as consultas de urgência que são atendidas no decorrer do dia.

A reunião de equipe e o atendimento noturno ocorrem semanalmente. A triagem por risco familiar é utilizada por algumas equipes da ESF, que realizam o levantamento das necessidades odontológicas através de questionário aplicado pelo Agente Comunitário de Saúde (ACS), além da utilização da ficha A que contem informações acerca da vulnerabilidade da clientela do território adscrito.

Nos dois modelos de saúde existentes o agendamento das consultas dos pacientes que necessitam de atendimento odontológico é realizado através das linhas de cuidado, que considera as condições sistêmicas dos pacientes (diabéticos DIA, hipertensos - HAS, DIA e HAS, gestantes), e os ciclos de vida (do bebê até o idoso).

A população alvo das ações de saúde bucal das UBS está centrada na área urbana, tendo cada unidade um território definido com uma média de 12.481 pessoas acima de dois anos de idade (faixa etária em que o cirurgião-dentista realiza o TCI). A UBS Planalto foi a responsável pela menor população, de 8.616 pessoas acima de dois anos, enquanto a unidade que apresentava em sua área o maior número de usuários na faixa etária apontada foi a da UBS Chico Mendes com uma população de 18.091 .

Nas USF o território de abrangência também é delimitado, entretanto existem unidades que prestam serviços para comunidades rurais, urbanas e mistas (rurais e urbanas), com a média de habitantes acima de dois anos de idade por unidade de 3.254, sendo que a USF Avencas/Flamingo apresentava o menor número de pessoas (1.816 usuários) e a que contava com maior número de cadastrados é a do Parque dos Ipês (3.784 pessoas acima de dois anos). A grande maioria das USF está implantada em áreas consideradas de risco no município como favelas, assentamentos, conjuntos habitacionais de casas populares e distritos.

Os dados coletados para observação foram levantados através dos relatórios gerenciais dos sistemas de informação, SIAB (Sistema de Informação da Atenção Básica) e Relatório de Avaliação de Saúde Bucal do Departamento de Coordenação de Saúde Bucal segundo cada unidade de saúde selecionada na amostra. O relatório de avaliação de Saúde Bucal da SMS forneceu o número de primeira consulta odontológica programática, o número de TCI, bem como o número de população $>$ de 2 anos e o SIAB a população total do território de cada unidade.
Foram elaborados instrumentos de coleta de dados que possibilitaram a observação e a avaliação dos dados analisando as unidades selecionadas diferenciadas por modelo de atenção a saúde apontando:

A totalização de TCI e Primeira consulta no período definido (2007 a 2009) por unidade, em um coeficiente por 100 habitantes;

População maior de dois anos, por unidade e o total de cada item do instrumento, por modelo de atenção;

Cálculo do percentual da resolutividade nas unidades de saúde, através do TCI como numerador e o TI como denominador x 100;

Para analisar a resolutividade dos diferentes modelos de atenção a saúde em relação aos dados da atenção básica (TCI e primeira consulta), realizou-se análise exploratória dos dados e, a seguir, foi aplicado o teste $t$ de Student para as variáveis que atenderam as pressuposições de uma análise paramétrica, com significância de 5\% e intervalo de confiança de $95 \%$. Os dados foram analisados utilizando-se o software SAS.

\section{Resultados}

A seguir, descrevem-se os resultados obtidos com o desenvolvimento do presente estudo, os quais serão apresentados através das Tabelas 1 e 2 .

Os resultados dos dados referentes às variáveis de primeira consulta odontológica (TI), que traduz o acesso ao tratamento, bem como a de tratamento completado inicial (TCI), que reflete a conclusão do iniciado, calculando-se o coeficiente por 100 habitantes por unidade de saúde analisada, estão descritos na Tabela 1.

As unidades que apresentaram os menores coeficientes para TI foram 7,3, 10,5 e 10,8/100 hab. para as UBS Chico Mendes, USF Jardim Cavalari e USF parque dos Ipês, respectivamente. Já a USF Figueirinha e a UBS JK que obtiveram o mesmo índice, foram as que apresentaram o maior coeficiente de TI, na ordem de 32,8/ 100 hab., acompanhadas logo em seguida pela USF Aeroporto com índice de 29,7/100 hab. e a USF Avencas Flamingo com 27,4/100 hab.

Ainda em relação à Tabela 1 , a unidade que apresentou o maior coeficiente em relação ao TCI foi a USF Avencas/Flamingo com resultado de 25,5/100 hab., seguida pela USF Figueirinha com 24,8/100 hab. e USF Rosália/Santa Helena com índice de 21,8/100 hab. A UBS Alto Cafezal foi a unidade com menor coeficiente de TCI, na ordem de 5,1/100 hab., seguida pela UBS Chico 
Tabela 1. Coeficiente de TI, TCI e resolutividade das unidades de atenção básica de Marília no período de 2007 a 2009.

\begin{tabular}{lrrrrrr}
\hline \multicolumn{1}{c}{ Unidades } & $\begin{array}{c}\text { População } \\
\text { estimada } \\
\text { > } \mathbf{2} \text { anos }\end{array}$ & TI & $\begin{array}{c}\text { Coeficiente } \\
\text { por 100 } \\
\text { hab. TI }\end{array}$ & TCI $\begin{array}{c}\text { Coeficiente } \\
\text { por 100 } \\
\text { hab. TCI }\end{array}$ & Resolutividade \\
\hline UBS Alto Cafezal & 12.984 & 2.204 & 16,9 & 673 & 5,1 & 30,5 \\
UBS Castelo Branco & 9.230 & 1626 & 17,6 & 937 & 10,1 & 57,6 \\
UBS Chico Mendes & 18.091 & 1.337 & 7,3 & 962 & 5,3 & 71,9 \\
UBS JK & 10.055 & 3.301 & 32,8 & 921 & 9,1 & 27,9 \\
UBS Planalto & 8.616 & 1.802 & 20,9 & 534 & 6,2 & 29,6 \\
UBS Bandeirantes & 10.109 & 2.094 & 20,7 & 594 & 5,8 & 28,3 \\
USF Aniz Badra & 3.113 & 547 & 17,5 & 375 & 12 & 68,5 \\
USF Rosália/Sta. Helena & 2.190 & 461 & 21 & 479 & 21,8 & 103,9 \\
USF Avencas/Flamingo & 1.816 & 499 & 27,4 & 464 & 25,5 & 92,9 \\
USF Figueirinha & 2.479 & 815 & 32,8 & 617 & 24,8 & 75,7 \\
USF Vila Barros & 2.760 & 508 & 18,4 & 357 & 12,9 & 70,2 \\
USF Aeroporto & 3.349 & 997 & 29,7 & 696 & 20,7 & 69,8 \\
USF Parque dos Ipês & 3.784 & 412 & 10,8 & 338 & 8,9 & 82 \\
USF Vila Hípica & 2.894 & 506 & 17,4 & 400 & 13,8 & 79 \\
USF Altaneira & 3.003 & 434 & 14,4 & 454 & 15,1 & 104,6 \\
USF Novo Horizonte/ Dirceu & 2.898 & 691 & 23,8 & 546 & 18,8 & 79 \\
USF Jardim Cavalari & 2.970 & 314 & 10,5 & 294 & 9,8 & 93,6 \\
\hline
\end{tabular}

Fonte: NI/SMS; PMSB/SMS; IBGE 2009

Tabela 2. Resultados da atenção básica, média (desvio padrão) em função da análise dos coeficientes de TI, TCI e resolutividade em saúde bucal no período de 2007 a 2009.

\begin{tabular}{lccc}
\hline & \multicolumn{2}{c}{ Grupos } & \\
\cline { 2 - 3 } \multicolumn{1}{c}{ Variável } & UBS & USF & p-valor \\
& média (dp) & média (dp) & \\
\hline TI coef/ 100hab & $19,37(8,24)$ & $20,34(7,41)$ & $0,8073 \mathrm{~ns}$ \\
TCI coef/100hab & $6,93(2,12)$ & $16,74(5,88)$ & $0,0014^{*}$ \\
Resolut Tci/Ti ${ }^{*} 100$ & $40,97(18,99)$ & $83,56(13,21)$ & $<0,0001^{*}$ \\
\hline
\end{tabular}

\# Teste $\mathrm{t}$ de Student; " significativo ( $\mathrm{p} \leq 0,05)$; ns não significativo ( $\mathrm{p}>0,05)$. TI: Tratamento Odontológico Inicial. TCI: Tratamento Completado Inicial.

Mendes, com 5,3/100 hab. e UBS Bandeirantes com resultado de 5,8/100 hab.

A análise da resolutividade aponta a USF Altaneira com o maior índice de resolutividade dentre todas as unidades integrantes da amostra, com o resultado de 104,6\%, seguida pela USF Rosália/Santa Helena com 103,9\%, à frente da USF Jardim Cavalari com 93,6\%. Esse resultado acima de $100 \%$ das unidades referidas se justifica observando que o tratamento odontológico dos pacientes possa ter sido iniciado antes de 2007 e concluído nos anos seguintes, se considerarmos o período de avaliação do presente estudo de 2007 a 2009.
As unidades com menor percentual de resolutividade foram a UBS Bandeirantes, com resultado de $28,3 \%$, seguida da UBS JK com 27,9\% e as UBS Planalto com 29,6\%.

Fica evidente que as unidades de saúde da família apresentaram melhores índices de resolutividade que as unidades tradicionais (confirmado na Tabela 2), sendo que cinco, das onze USF analisadas, atingiram mais que $80 \%$ de resolutividade, e a UBS que obteve o maior índice, a UBS Chico Mendes, não ultrapassou o percentual de $72 \%$, que é muito próximo ao resultado obtido pela USF com menor índice de resolutividade dentre as USF, a USF Aniz Badra, que apre- 
sentou a resolutividade de $68,5 \%$. Das seis UBS analisadas, quatro apresentaram índice de resolutividade abaixo de $50 \%$.

Ressalta-se a UBS Chico Mendes em relação as outras unidades básicas de saúde, com maior índice de resolutividade haja vista que comparada às outras unidades iniciou um número menor de tratamentos, conseguindo efetivamente concluir mais tratamentos.

Na Tabela 2 são apontadas as médias para os coeficientes de TI, TCI e resolutividade das unidades de saúde por modelo de atenção, USF e UBS.

Para o coeficiente TCI/pop há uma diferença estatisticamente significativa entre os grupos (UBS e USF), o que espelha a diferença encontrada, onde verifica-se $40,97 \%$ de resolutividade nas UBS e $83,56 \%$ nas USF. Portanto, fica evidente que o modelo USF apresenta maior resolutividade que o modelo UBS com uma diferença de mais de 40 pontos percentuais.

\section{Discussão}

É importante destacar que a literatura praticamente não apresenta trabalhos que analisam a resolutividade das ações de saúde bucal em atenção básica, não havendo, desta forma, resultados expressos para comparação aos obtidos neste estudo, sendo que os trabalhos que analisam modelos em saúde bucal se restringem a avaliação de um serviço ou programa específico, de diagnóstico da situação de serviços de saúde, estudos de caso sobre a implementação de um programa ou política pública ${ }^{15,16}$.

Inicialmente, observa-se que os coeficientes relativos ao TI não foram estatisticamente diferentes. Já no que se refere ao coeficiente TCI sobre a população estimada maior que dois anos, verificam-se valores de 16,74 para o modelo USF e 6,93 para o modelo UBS, havendo uma diferença estatisticamente significativa e que espelha a diferença de $40,97 \%$ de resolutividade na UBS em relação aos $83,56 \%$ da USF.

Portanto, de acordo com os dados estatísticos, fica evidente que o modelo USF apresenta maior resolutividade que o modelo UBS com uma diferença de mais de $40 \%$ em relação à conclusão de tratamentos odontológicos iniciados. O impacto das ações de saúde bucal no PSF tem evidente progresso do ponto de vista da universalidade, equidade e integralidade ${ }^{17}$, entretanto não existe comparação com outro modelo, portanto acredita-se ser uma análise peculiar deste estudo.
Quando comparada ao modelo Saúde da Família como um todo no que se refere ao impacto das ações, um estudo realizado em 2006 pela Universidade Estadual de Pelotas comparou a atenção básica tradicional com a Saúde da Família e mostrou que o cuidado de idosos foi maior nas áreas atendidas pela SF, comparativamente à atenção prestada pelas unidades tradicionais ${ }^{18}$.

Em relação à análise dos indicadores de saúde geral como a mortalidade infantil, indicador de avaliação mundial das ações da APS e qualidade de vida, um estudo sobre a avaliação do impacto da ESF sobre a Mortalidade Infantil no período de 1990 a 2002, identificou que o aumento da cobertura populacional pela Saúde da Família teve o maior impacto na redução da mortalidade infantil, sendo que a cada $10 \%$ de população coberta houve a redução maior que o mesmo percentual de acesso a água tratada, ou que o mesmo percentual de expansão do acesso à leitos hospitalares ${ }^{18}$.

Desta forma, a significativa diferença apontada neste estudo, pode estar associada ao modo singular de implantação e o propósito da Saúde da Família onde a equipe de saúde bucal deve ter a responsabilidade de criar e manter vínculo entre a comunidade e os profissionais de saúde, garantindo assim a longitudinalidade do cuidado. Esta afirmativa é ressaltada pelas USF que atingiram elevados índices (acima de 100\% de resolutividade) estando estes resultados relacionados diretamente ao perfil profissional do cirurgião-dentista juntamente ao envolvimento com o restante da equipe de saúde, com ênfase no compromisso e organização do trabalho conforme as diretrizes estabelecidas para a Saúde da Família.

Os autores deste estudo concordam com Mendes ${ }^{19}$ que considera o cuidar como mais que vigiar, portanto é, também, ampliar o conceito de vigilância à saúde bucal, sendo que a Saúde da Família propicia esta aplicação por intervir com a família num relacionamento cooperativo em que a equipe de saúde procura aliar os conhecimentos científicos às habilidades de observação, comunicação e intuição. A atuação não fica restrita à espera dos sinais de alarme ou da ocorrência de eventos sentinela, mas a detecção precoce de estrangulamentos, ou de nós críticos que evidenciam os problemas e as necessidades de saúde.

Já em relação ao modelo instituído na UBS, as ações em saúde bucal estão focadas sobremaneira na atenção à demanda espontânea, sendo que os tratamentos odontológicos iniciados, geralmente são em usuários que demandam o serviço com alguma queixa odontológica, onde é 
identificada a necessidade de tratamento e a proposição de continuidade para conclusão das necessidades individuais em saúde bucal, reforçada pela lógica instituída no processo histórico de construção do cuidado em saúde no país, no que se refere à atenção produzida pelo modelo biomédico, atuando com foco na cura de doenças, que ainda é hegemônica nas práticas dos serviços de saúde ${ }^{20}$.

Isto se justifica pelos baixos resultados de resolutividade identificados nas UBS, tendo o maior índice não ultrapassado $72 \%$, o que está intimamente relacionado com o processo de trabalho e perfil dos profissionais dos cirurgiões-dentistas e equipe de saúde, que na grande maioria exercem práticas tradicionais de longa data, o que provavelmente impacta na organização do processo de trabalho focado na lógica historicamente adotada na atenção em saúde bucal no país, além do vasto território adscrito à unidade de saúde dificultando o acompanhamento mais próximo dos usuários.

Outro ponto importante a ser discutido que pode contribuir para o entendimento dos resultados obtidos neste trabalho, em relação à resolutividade significantemente maior da Saúde da Família frente à UBS, é a organização do processo de trabalho, sendo que sobre o modo de assistir as pessoas, a produção do cuidado, que requer vínculo, divisão de responsabilidades e resolutividade, está diretamente ligada à concepção de trabalho e à subjetividade da cada profissional e usuário, e pode impactar diretamente no processo de produção em saúde e cuidado das pessoas $^{21}$. Embora na UBS Chico Mendes a resolutividade tenha atingido valores próximo a da USF, justificado pela melhor organização do processo de trabalho na unidade, com adequado controle de vagas e faltosos, havendo priorização para conclusão dos tratamentos odontológicos iniciados.

No presente estudo, pode-se dizer que há uma diferença considerável em relação à organização do processo de trabalho entre os dois modelos de acordo com as características peculiares que descrevem cada um.

Na UBS, a população adscrita à área de abrangência é bem superior à da USF, dificultando o acesso territorial geográfico dos pacientes para a unidade de saúde até o reconhecimento de toda a clientela do território e, consequentemente, a formação de vínculo e adesão ao tratamento, já que muitos pacientes iniciam este por meio da consulta odontológica programática (TI) e concluise efetivamente poucos tratamentos (TCI), com- prometendo assim o pressuposto do acompanhamento longitudinal almejado para a APS com impacto direto sobre a resolutividade da saúde bucal neste modelo de atenção.

É importante ressaltar ainda que a avaliação dos índices utilizados para obtenção do resultado das unidades de saúde analisadas neste estudo, de forma separada ou única, TI e TCI não refletem em impacto na resolutividade, haja vista que mesmo a unidade apresentando alto coeficiente de TI, a resolutividade pode ser baixa, caso o coeficiente de TCI seja baixo. Portanto, a análise do TI isoladamente mede o nível de acessibilidade ao tratamento odontológico, mas não reflete o grau de resolutividade, assim como o TCI analisado separadamente, apenas traduz a conclusão de tratamentos odontológicos na unidade de saúde a cada 100 habitantes.

Percebe-se que a maioria dos trabalhos encontrados na literatura em relação à avaliação de modelos de atenção faz uma dicotomia entre a organização do processo de trabalho da equipe de saúde bucal com a do modelo de saúde geral existente no lócus de desenvolvimento da ação, a unidade de saúde. Assim, o entendimento é que o modelo geral é o que prevalece como indutor da organização das ações de saúde bucal conforme o macro modelo organizacional existente na unidade de saúde. Além de praticamente inexistência de trabalhos voltados à avaliação das ações de saúde bucal em relação ao modelo organizativo do processo de trabalho, apenas um estudo comparou, até o presente momento, as modalidades assistenciais do PSF e UBS por estrato de exclusão social, considerando as opiniões de usuários, profissionais de saúde e gestores, e não observou diferenças significativas para profissionais e gestores ${ }^{22}$. Contudo, o índice de atenção básica, aferido por meio dos usuários, foi mais elevado nas unidades PSF do que nas UBS, o que corrobora com os resultados obtidos no presente estudo onde a ESF se destacou com maior resolutividade em relação à UBS.

Desta forma, nos poucos estudos existentes na literatura sobre a comparação entre os modelos de atenção, encontra-se divergência nas conclusões obtidas, tornando evidente que existem variáveis diversas a serem consideradas na efetiva identificação de qual modelo é melhor, haja vista as peculiaridades do local em que se está efetivando o estudo ${ }^{23,24}$.

Podemos pensar como possível limitação do estudo, o seu caráter local, contudo parece lógico acreditarmos que além da discussão do modelo de gestão envolvido na questão da resolutivida- 
de, o processo de trabalho também permeia as características do sistema de saúde. Desta forma, os resultados deste estudo poderiam ser inferidos a outras localidades, obviamente levando em consideração as peculiaridades dos sistemas locais, sendo importante instrumento para os gestores de saúde avaliar os modelos de atenção operantes.

\section{Considerações finais}

Conclui-se que o tipo de modelo assistencial adotado no município de Marília interfere na resolutividade das ações de saúde bucal na atenção bá- sica, sendo melhor para o modelo baseado na Saúde da Família, evidenciando impacto efetivo nos resultados de acesso (TI), conclusão de tratamento odontológico iniciado (TCI) e resolutividade na atenção básica, onde a Saúde da Família se mostrou melhor no coeficiente de TCI e resolutividade frente à UBS.

Desta forma, é importante avaliar os modelos assistenciais de saúde bucal estruturados com a finalidade de orientar o planejamento e a execução de ações na esfera municipal indispensável para a construção de uma odontologia pública mais resolutiva, de maior eficácia e qualidade, fazendo concretizar para a população os princípios do SUS.

\section{Colaboradores}

J Bulgareli, KL Cortellazzi, GMB Ambrosano, MC Meneghim, ET Faria, FL Mialhe e AC Pereira participaram igualmente de todas as etapas de elaboração do artigo. 


\section{Referências}

1. Brasil. Conselho Nacional dos Secretários de Saúde (CONASS). Atenção Primária e promoção da Saúde. Brasília: CONASS; 2007.

2. Sampaio LFR, Souza HM. Atenção Básica: política. Diretrizes e modelos coletâneos no Brasil. In: Negri B, Faria R, Viana A, organizadores. Recursos Humanos em Saúde. Política, desenvolvimento e mercado de trabalho. São Paulo: Unicamp; 2002. p. 9-32.

3. Brasil. Ministério da Saúde (MS). Portaria no 648 , de 28 de março de 2006. Aprova a Política Nacional de Atenção Básica, estabelecendo a revisão de diretrizes e normas para a organização da Atenção Básica para o Programa Saúde da família (PSF) e o Programa Agentes Comunitários de Saúde (PACS). Diário Oficial da União 2006; 29 mar.

4. Brasil. Ministério da Saúde (MS). Secretaria de Assistência à Saúde. Departamento de Descentralização da Gestão da Assistência. Regionalização da Assistência à Saúde: aprofundando a descentralização com eqüidade no acesso: Norma Operacional da Assistência à Saúde: NOAS-SUS 01/02 e Portaria MS/GM n. ${ }^{\circ} 373$, de 27 de fevereiro de 2002 e regulamentação complementar. Brasília: MS; 2002.

5. Madureria PR, Capitani, EM, Campos GWS. Avaliação da qualidade da atenção à saúde na rede básica. Cad Saude Publica 1989; 5(1):45-59.

6. Starfield B. IS US health really the Best in the world. JAMA 2000; 284(4):483-485.

7. Calado GS. A inserção de equipe de saúde bucal no programa de saúde da família: principais avanços e desafios [tese]. Rio de Janeiro: Escola Nacional de Saúde Pública (ENSP), Fundação Oswaldo Cruz (Fiocruz); 2002.

8. Junqueira SR, Frias AC, Zilbovicius C. Saúde bucal coletiva: quadros social, epidemiológico e político. In: Rode SM, Nunes SG, organizadores. Atualização clínica em odontologia. São Paulo: Artes Médicas; 2004. p. 591-604.

9. Botazzo C, Oliveira MA. Atenção Básica no Sistema Único de Saúde: abordagem interdisciplinar para os serviços de saúde bucal. São Paulo: Páginas \& Letras Editora e Gráfica; 2008.

10. Brasil. Ministério da Saúde (MS). Portaria no 91, de 10 de janeiro de 2007. Regulamenta a unificação do processo de pactuação de indicadores e estabelece os indicadores do Pacto pela Saúde, a serem pactuados por Municípios, Estados e Distrito Federal. Diário Oficial da União 2007; 11 jan.

11. Brasil. Ministério da Saúde (MS). Aprova o Manual para organização da Atenção Básica no Sistema único de Saúde. Portaria n. 3.925 de 17 de novembro de 1998. Diário Oficial da União 1999; 2 fev.

12. Gonçalves RBM. Tecnologia e organização social das práticas de saúde: características tecnológicas do processo de trabalho na rede estadual de centros de saúde de São Paulo. São Paulo: Hucitec; 1994.
13. Instituto Brasileiro de Geografia e Estatística (IBGE). Pesquisa Nacional por Amostra de Domicílios 2011. v. 29. [documento na Internet]. [acessado 2013 nov 27]. Disponível em: ftp://ftp.ibge.gov.br/Trabalho _e_Rendimento/Pesquisa_Nacional_por_Amostra _de_Domicilios_anual/2011/Sintese_Indicadores/ sintese_pnad2011.pdf

14. Brasil. Portaria no 648/GM, de 28 de março de 2006. Aprova a Política Nacional de Atenção Básica, estabelecendo a revisão de diretrizes e normas para a organização da Atenção Básica para o Programa Saúde da Família (PSF) e o Programa Agentes Comunitários de Saúde (PACS). Diário Oficial da União 2006; 29 mar.

15. Almeida NS, Araújo MVA, Araújo IC. Atendimento odontológico no SUS: manual para o cirurgião-dentista. Medcenter.com Odontologia [acesso 2009 maio 28]. Disponível em: http://dtr2004.saude.gov.br/ dab/documento/inforrmes/psfinfo9.pdf.

16. Nickel DA. Modelo de avaliação da efetividade da atenção em saúde bucal [dissertação]. Florianópolis: Universidade Federal de Santa Catarina; 2008.

17. Carvalho DQ, Ely HC, Paviani LS, Corrêa PEB. A dinâmica da equipe de saúde bucal no Programa Saúde da Família. Boletim da saúde 2004; 18(1):175184.

18. Brasil. Ministério da Saúde (MS). Painel de Indicadores do SUS 4. Temático Saúde da Família. Brasília: MS, Organização Pan-Americana da Saúde (OPAS); 2008.

19. Mendes EV. Uma agenda para a saúde. São Paulo: Hucitec; 1999.

20. Morosini MVGC, Corbo ADA, organizadores. Modelos de atenção e a saúde da família. Rio de Janeiro: EPSJV, Fiocruz; 2007.

21. Franco T, Merhy E. PSF: contradições e novos desafios. Conferência Nacional de Saúde On-line; 1999 [acessado $2010 \mathrm{fev}$ 3]. Disponível em: http://www. datasus.gov.br/cns/cns.htm.

22. Elias PE, Ferreira CW, Alves MCG, Cohn A, Kishima V, Escrivão Júnior A, Gomes A, Bousquat A. Atenção Básica em Saúde: comparação entre PSF e UBS por estrato de exclusão social no município de São Paulo. Cien Saude Colet 2006; 11(3):633-641.

23. Lentsck MH, Kluthcovsky ACGC, Kluthcovsky FAK. Avaliação do Programa Saúde da Família: uma revisão. Cien Saude Colet 2010; 15(Supl. 3):3455-3466.

24. Silvia LA, Casotti CA, Chaves SCL. A produção científica brasileira sobre a Estratégia Saúde da Família e a mudança no modelo de atenção. Cien Saude Colet 2013; 18(1):221-232.

Artigo apresentado em 31/10/2012

Aprovado em 10/05/2013

Versão final apresentada em 23/05/2013 
